\title{
THE CAUCHY RELATIONS IN A MOLECULAR THEORY OF ELASTICITY*
}

\author{
BY \\ IVAR STAKGOLD \\ Cruft Laboratory, Harvard University
}

1. Introduction and summary. If an elastic body is considered as a continuum, the existence of a "strain energy density" can be ascertained.

Using a molecular theory of elasticity, we can deduce the existence of an energy function of the internal forces between particles. It will be shown that this energy function can be expressed as a function of an adequately defined strain. In the case of a monatomic crystalline body, this energy function corresponds to the strain energy density of a continuous body. For a multiatomic crystalline body, an analogy with the strain energy of a continuous body can be drawn.

But in all these cases, it will finally be possible to write the energy density as:

$$
\phi=\phi\left(e_{i j}\right) .
$$

We can then assume the existence of a suitably convergent series expansion for $\phi\left(e_{i j}\right)$,

$$
\begin{aligned}
\phi & =\phi_{0}+\Omega^{i i} e_{i j}+\Lambda^{i j, h k} e_{i j} e_{h k}+\cdots, \\
& =\phi_{0}+\phi_{1}+\phi_{2}+\cdots
\end{aligned}
$$

(see [1] $\nmid$ p. 230). The usual summation convention for an index appearing once covariantly and once contravariantly is used here. The coefficients $\Lambda^{i i, h k}$ of the quadratic term in the expansion are those involved in the so-called "Cauchy relations."

We observe that $\Lambda^{i j, h k}$ is a three-dimensional tensor of the fourth rank, and, as such, can have up to 81 independent components. By inspection of (1.1), we notice a number of restrictions on $\Lambda^{i j, k t}$ which immediately reduce the number of independent components.

Let us call $(i, j)$ the indices of the first group and $(h, k)$ the indices of the second group. It is clear that we can choose the coefficients $\Lambda^{i j, h k}$ to be symmetric in the two groups, i.e.,

$$
\Lambda^{i, h k}=\Lambda^{h k, i j} .
$$

Since $e_{i j}$ is a symmetric tensor, it is also evident that we can choose $\Lambda^{i j, h k}$ to be symmetric with respect to indices of both the first and second group. Hence:

$$
\Lambda^{i, h k}=\Lambda^{i i, h k}=\Lambda^{j i, k h}=\Lambda^{i j, k h}=\cdots .
$$

Taking into account these additional conditions on $\Lambda$, we conclude that only 21 independent components remain from the original 81.

The Cauchy relations are not included in (1.2) nor in (1.3), but consist of six additional relations

$$
\Lambda^{i i, h k}=\Lambda^{i h, i k}=\Lambda^{i k, h i}=\cdots
$$

*Received Jan. 25, 1949.

$\dagger$ Numbers in the brackets refer to the bibliography at the end of the paper. 
whose validity we are to investigate. In other words, we are to find out whether $\Lambda^{i i, h k}$ is completely symmetric or only partially symmetric to the extent given by (1.2) and (1.3).

This formulation of the problem was given by Brillouin ([1], p. 234) and previously suggested by Voigt ([5], p. 607) and Born ([2], p. 554).

Within the framework of a continuum theory, no relations of the Cauchy type can be arrived at. It is only when we picture the elastic body as a set of particles with interacting forces that we can hope to arrive at the Cauchy relations. Naturally, we expect these relations to hold only under certain specific assumptions as to the type of body and the type of interacting forces.

It is the purpose of this paper to discover the assumptions necessary (or sufficient) to insure the validity of the Cauchy relations.

2. Historical summary of work on the Cauchy relations. As has been pointed out previously, all the investigations on the Cauchy relations must be based on a molecular theory of elasticity.

Born was the first (at least to the author's knowledge) to discover the fundamental distinction between the monatomic and the multiatomic cases. All previous authors had assumed that the concept of strain, as defined for a continuum, could be applied without any modifications to a molecular body. Born proves conclusively that this transfer is permissible only for a monatomic body, where each particle is a center of symmetry. He shows that the concept of a continuous strain could not be used directly for the multiatomic body. The other authors discussed in this chapter do not make this distinction, and their work is, therefore, only applicable to monatomic bodies.

The Cauchy relations were claimed by Cauchy to be valid for the case of a molecular body in which the interactions between particles are central forces. Many writers have verified the Cauchy relations in the case of central forces and have shown that these relations are not valid if the interaction forces are not central (see [2], p. 555; [3], pp. 47-49; [5], pp. 607, 608). All these verifications (except Poincaré's) are based on a linear approximation to the strain tensor, rather than the correct expression for the strain tensor. The error, thereby introduced, will be shown to be of the order of magnitude of the remaining terms.

The method of attack used by the majority of authors (including Voigt and Born) involves a series expansion for the energy function $\phi$ in terms of the Cartesian displacement components of the individual particles. After such an expansion is obtained, the displacement is expressed in terms of the local strain and is then substituted in the expansion. The resulting series for $\phi$ is a development in terms of the strain. The coefficient of the quadratic term is examined to ascertain the fulfillment of the Cauchy relations.

The difficulty of the method lies in expressing the displacement in terms of the strain. This involves the solution of the set of non-linear partial differential equations of the first order,

$$
e_{i j}=\frac{1}{2}\left(\frac{\partial s_{i}}{\partial y^{j}}+\frac{\partial s_{j}}{\partial y^{i}}\right)+\frac{1}{2} \sum_{k} \frac{\partial s_{k}}{\partial y^{i}} \frac{\partial s_{k}}{\partial y^{j}}
$$

Within the region of appreciable molecular interaction, it can be assumed that $e_{i i}$ is constant. 
In the one-dimensional case, $(2.1)$ can readily be solved:

$$
s=(1+2 e)^{1 / 2} x-x,
$$

which reduces to $s=e x$ for very small strain $e$. It is, therefore, possible that a closed solution to (2.1) might exist. The exact solution must have appeared too laborious to writers on the topic, for no recorded attempt at an exact solution can be found in the literature.

Born and Voigt neglect the quadratic term in the derivatives in (2.1) and arrive at

$$
s_{i}=e_{i} y^{i} .
$$

Expression (2.3) is substituted in the series for the energy density $\phi$. Born and Voigt then conclude that, in the case of zero initial external forces, the Cauchy relations are satisfied by the coefficient of the quadratic term in the strains.

Epstein, in 1946 (see [4]) presented a justified objection to the approximation (2.3) used by Born. He states that (2.1) must be solved up to terms quadratic in $e_{i}$, to insure derivation of the complete quadratic term in the strain series expansion for $\phi$. He points out that this more delicate approximation need only be substituted in $V_{1}$, the linear term in the displacement series for $\phi$. In the quadratic term in the displacement series, the Born formula (2.3) may be substituted.

There can be little question as to the propriety of Epstein's observation. Unfortunately, he uses an unnecessarily 'complicated method to arrive at the conclusion that the Cauchy relations are satisfied by the coefficient of the complete quadratic term in the strain series for $\phi$. Then Epstein states that the "coefficients of elasticity" do not satisfy the Cauchy relations. He considers the case where the initial external forces are zero. In this case, $V_{1}=0$, hence the energy is given by the term quadratic in the displacement $\left(V_{2}\right)$. He then states that this term is not equal to the entire quadratic term $E_{2}$ of the strain series, and that, in addition, $V_{2}$ does not satisfy the Cauchy relations.

In the case of zero initial external forces, the only case which Epstein considers, we will show that $E_{2}$ and $V_{2}$ are equal and that the coefficients appearing in them are also equal. We will also show that, from a theoretical point of view, it is much more logical to define the coefficients of elasticity from $E_{2}$ than from $V_{2}$. Epstein does not notice that $E_{2}$ and $V_{2}$ are equal. As a matter of fact, he explicitly states that his entire argument rests on the fact that they are not equal.*

To arrive at our results, we will use a method of attack different from that of the majority of writers on the subject. This method has several advantages: it lends itself to simpler and more rigorous treatment; it does not involve approximations to the solution of partial differential equations; and it presents a clear tensorial outlook. The tensorial characteristics can be recognized immediately and all invariant relationships become apparent. These advantages are achieved by using, almost from the start, a series for $\varphi$ in terms of the strain rather than a series in terms of the displacement.

We will resort only to approximations of a physical nature, based on universally-

*This has been pointed out by Zener (Phys. Rev. 71, 323 (1947)). A somewhat more detailed analysis is given by Per Olov Löwdin (A theoretical investigation into some properties of ionic crystals, Upsala, 1948). This latter pamphlet discusses the Cauchy relations in the case of finite strains and arrives at conclusions entirely in agreement with those in Sec. 4. The pamphlet reached the United States in the fall of 1948, after this thesis had been completed. 
recognized experimental facts. These approximations are part of all other treatments of this subject, although the various authors have not always stated them clearly.

3. A summary of a few well-known results of the theory of elasticity for a continuum. Let the original coordinates of a point (i.e. before deformation) be represented by $a^{i}$. The final or strained coordinates will be denoted by $x^{i}$. The coordinate systems need not be the same.

Let $d s_{0}^{2}$ denote the square of the unstrained distance between two neighboring points; let $d s^{2}$ denote the square of the distance between the same two points after deformation. We then introduce the Lagrangean strain tensor $e_{i j}$ for the deformation of a continuous body; from its definition

$$
d s^{2}-d s_{0}^{2}=2 e_{i j} d a^{i} d a^{i} .
$$

The existence of a strain energy representing the potential energy of the deformation has been shown by many writers. (e.g., see [1], p. 229).

Let $E$ be the total strain energy for a continuous body. We write

$$
E=\int_{V} \varphi d V
$$

where $\phi$ is the strain energy density and $d V$ is an element of volume in the unstrained coordinates.

Since the strain $e_{i j}$ completely determines the energy of the body, it is clear that $\phi=\phi\left(e_{i j}\right)$. We assume that

$$
\varphi=\varphi_{0}+\varphi_{1}+\varphi_{2}+\cdots=\varphi_{0}+\Omega^{i i} e_{i i}+\Lambda^{i j, h k} e_{i j} e_{h k}+\cdots .
$$

Now $\varphi$ is a scalar, as are $\varphi_{0}, \varphi_{1}, \varphi_{2}$, etc. Hence $\Omega^{i j}$ is a doubly contravariant symmetric tensor, and $\Lambda^{i j, h k}$ is a four times contravariant tensor symmetric in $(i, j)$ and in $(h, k)$. The two groups $(i j),(h k)$ are also interchangeable. No additional symmetry in this tensor can be discovered by treating the body as a continuum. Hence the Cauchy relations are not satisfied.

4. The monatomic lattice. Consider a simple monatomic lattice. All the particles are similar and have the same force law between them. We will assume, for the present, that knowledge of the position of every particle in the body completely determines the energy of the body. We are excluding the possibility of dipole moments attached to the particles; in this case, the energy would also depend on the angle between the dipole vector and some reference frame. It may seem that we are also excluding the monatomic lattice in which the particles are considered as rigid bodies with ability to rotate. It was shown by Born that this case reduces to the case of a multiatomic lattice (see [2], p. 556), which we will treat in Sec. 5 .

We conclude then that the total internal energy of the body depends only on the distances between atoms. This is not equivalent to the central force assumption, where the total energy is the sum of mutual energies between pairs of particles, this mutual mutual energy being a function only of the distance between the two particles on which it acts.

Let $\mu, \nu, \eta, \cdots$ be particles of the body, $\rho_{\mu \nu}$ the distance between the particles $\mu$ and $\nu, E$ the energy function for the internal forces. It will be more convenient for our 
purposes to use $R_{\mu \nu}=\rho_{\mu \nu}^{2}$ as the independent variable; this is permissible since $\rho_{\mu \nu}$ is positive.

$$
E=E\left(R_{\mu \nu}, R_{\mu \eta}, R_{\eta \nu}, \cdots\right) \text {, in general. }
$$

The case of central forces occurs when the function $E$ can be decomposed into

$$
E=\frac{1}{2} \sum_{\mu, \nu} E_{\mu \nu}\left(R_{\mu \nu}\right), \quad \text { for central forces. }
$$

(In (4.2) $R_{\mu \nu}, R_{\nu \mu}$ are treated as independent variables.)

Expanding (4.1) in a power series, we get

$$
\begin{aligned}
E=E_{0}\left(R_{\mu \nu}^{0}, R_{\mu \eta}^{0}, \cdots\right) & +\sum_{\mu, \nu}\left(\frac{\partial E}{\partial R_{\mu \nu}}\right)_{0} \Delta R_{\mu \nu} \\
& +\frac{1}{2} \sum_{\mu, \nu ; \eta, \xi}\left(\frac{\partial^{2} E}{\partial R_{\mu \nu} \partial R_{\eta \xi}}\right)_{0} \Delta R_{\mu \nu} \Delta R_{\eta \xi}+\cdots,
\end{aligned}
$$

where the summation is over all pairs of particles $\mu, \nu$. We may take $E_{0}=0$ as our datum.

Let us divide the body into regions $V, V^{\prime}, \cdots$. We define the energy of $V$ as

$$
E_{V}=\sum_{\substack{\mu, \nu \\ \text { in } V}}\left(\frac{\partial E}{\partial R_{\mu \nu}}\right)_{0} \Delta R_{\mu \nu}+\frac{1}{2} \sum_{\substack{\mu, \nu ; \eta, \xi \\ \text { in } V}}\left(\frac{\partial^{2} E}{\partial R_{\mu \nu} \partial R_{\eta \xi}}\right)_{0} \Delta R_{\mu \nu} \Delta R_{\eta \xi}+\cdots .
$$

We notice that $E \neq \sum_{V} E_{V}$, but that we have

$$
E \cong \sum_{\mathrm{a} 11 \mathrm{~V}} E_{V}
$$

It is shown in the appendix that the equality sign in (4.5) becomes valid as long as each volume $V$ has dimensions which are large compared with the distance of appreciable molecular interaction.

Let us now consider a continuum superposed on our monatomic lattice before deformation. Each particle $\mu_{0}$ will have a coinciding representation $\mu_{c_{0}}$ on the continuum. The continuum upon deformation will have a strain $e_{i j}$, which is a continuous function of the coordinates. Hence, within a small volume $V$ (which can still contain a very large number of representations $\mu_{c}$ ), the strain is sensibly homogeneous.

For a monatomic lattice, equilibrium is achieved in the unstrained state by having each particle a center of symmetry (the laws of force between all particles are the same). Are we permitted to assume that, after deformation, the particles still coincide with their representations on the deformed continuum? The only way the assumption can fail is by causing violation of the equilibrium of individual particles after deformation. But under the proposed assumption, each particle $\mu$ will still be a center of symmetry after deformation (at least with respect to the nearby particles, which are the only ones influencing the force on $\mu$ ). Hence, we conclude that the assumption is permissible for a monatomic lattice, since equilibrium of all particles is maintained after deformation.

Consider a volume $V$ whose dimensions are small macroscopically speaking, but very large compared with the distance of appreciable molecular interaction. (From our knowledge of the forces of interaction, such volumes exist.) The assumption of the existence of such a volume $V$ about each particle of the body is necessary to treat 
inhomogeneous strain (this assumption is made by all authors, although not always stated).

Within $V, e_{i j}$ is nearly constant. From the definition of $e_{i j}$, we have

$$
\Delta R_{\mu \nu}=2 e_{i i} a_{\mu \nu}^{i} a_{\mu \nu}^{i}, \quad \text { if } \mu, \nu \text { are close together }
$$

( $e_{i j}$ is the Lagrangean strain tensor; $a_{\mu \nu}^{i}=a_{\nu}^{i}-a_{\mu}^{i}$, where $a_{\mu}^{i}$ are the unstrained coordinates of the point $\mu$ ). Since $V$ is a small volume on a macroscopic scale, (4.6) holds for any two particles of $V$.

Substituting (4.6) into (4.4) we obtain

$$
E_{V}=\sum_{\substack{\mu, \nu \\ \text { in } V}}\left(\frac{\partial E}{\partial R_{\mu \nu}}\right)_{0} 2 e_{i j} a_{\mu \nu}^{i} a_{\mu \nu}^{i}+\frac{1}{2} \sum_{\substack{\mu, \nu ; \eta, \xi \\ \text { in } V}}\left(\frac{\partial^{2} E}{\partial R_{\mu \nu} \partial R_{\eta \xi}}\right)_{0} 4 e_{i j} e_{h k} a_{\mu \nu}^{i} a_{\mu \nu}^{i} a_{\eta \xi}^{h} a_{\eta \xi}^{k}+\cdots
$$

We are now in a position to define the strain energy density $\varphi=\lim _{V \rightarrow 0}\left(E_{V} / V\right)$. The limit is taken macroscopically, so that the smallest volume still contains a very large number of particles:

$\varphi=\frac{1}{V}\left[\sum_{\substack{\mu, \nu \\ \text { in } V}}\left(\frac{\partial E}{\partial R_{\mu \nu}}\right)_{0} 2 e_{i j} a_{\mu \nu}^{i} a_{\mu \nu}^{i}+\frac{1}{2} \sum_{\substack{\mu, \nu ; \eta, \xi \\ \text { in } V}}\left(\frac{\partial^{2} E}{\partial R_{\mu \nu} \partial R_{\eta \xi}}\right)_{0} 4 e_{i j} e_{h k} a_{\mu \nu}^{i} a_{\mu \nu}^{i} a_{\eta \xi}^{h} a_{\eta \xi}^{k}+\cdots\right]$.

Now (4.8) is a series of the type (1.1) and we immediately pick out

$$
\Lambda^{i j, h k}=\frac{2}{V}\left[\sum_{\substack{\mu, \nu ; \eta, \xi \\ \text { in } V}}\left(\frac{\partial^{2} E}{\partial R_{\mu \nu} \partial R_{\eta \xi}}\right)_{0} a_{\mu \nu}^{i} a_{\mu \nu}^{i} a_{\eta \xi}^{h} a_{\eta \xi}^{k}\right],
$$

where $\left(\partial^{2} E / \partial R_{\mu \nu} \partial R_{\eta \xi}\right)_{0}$ is a scalar in the unstrained coordinates. In general, $\Lambda^{i i, h k}$ will not satisfy the Cauchy relations, since indices of the first group are not interchangeable with indices of the second group. For instance, $i$ and $h$ cannot be interchanged in (4.9).

If we assume central forces, then substituting in (4.7), we get:

$$
\begin{gathered}
E=\frac{1}{2} \sum_{\mu, \nu} E_{\mu \nu}\left(R_{\mu \nu}\right), \\
E_{V}=\sum_{\substack{\mu, \nu \\
\text { in } V}}\left(\frac{d E_{\mu \nu}}{d R_{\mu \nu}}\right)_{0} e_{i j} a_{\mu \nu}^{i} a_{\mu \nu}^{i}+\sum_{\substack{\mu, \nu \\
i \text { in } V}}\left(\frac{d^{2} E_{\mu \nu}}{d R_{\mu \nu}^{2}}\right)_{0} e_{i j} e_{h k} a_{\mu \nu}^{i} a_{\mu \nu}^{i} a_{\mu \nu}^{h} a_{\mu \nu}^{k}+\cdots,
\end{gathered}
$$

and

(for central forces)

$$
\Lambda^{i i, h k}=\frac{1}{V} \sum_{\substack{\mu, \nu \\ \text { in } V}}\left(\frac{d^{2} E_{\mu \nu}}{d R_{\mu \nu}^{2}}\right)_{0} a_{\mu \nu}^{i} a_{\mu \nu}^{i} a_{\mu \nu}^{h} a_{\mu \nu}^{k} .
$$

It is apparent that the Cauchy relations are satisfied, since complete symmetry in $i, j$, $h, k$ exists.

It should be noted that no approximation to the strain tensor is made throughout the proof. The strain tensor is introduced through its very definition. The only approximation used is one which is used by all other writers, i.e., about each internal point of the body there exists a region whose dimensions are large compared with ranges of atomic interaction, but in which the strain can be treated as homogeneous. 
It is also well to remark that the coordinate system used in the proof is not specified and need not be a Cartesian system. Hence it can be concluded that (4.9) and (4.11) are, indeed, general tensor formulas.

Having arrived at the desired results, we are now in a position to compare our work with that of other authors on the subject. There have been so many authors who have written on the subject that it will be impossible to discuss the investigations of all of them. The classical proof of Cauchy's relation is well given in Born ([2], pp. 545-555). We shall limit ourselves to a discussion of his work. In addition, we will look into the work of Epstein as presented in his article, which was one of the impetuses for the writing of this thesis. Since these authors restrict themselves to central forces, we will write

$$
E_{V}=\sum_{\substack{\mu, \nu \\ \text { in } V}} \frac{1}{2}\left(\frac{d E_{\mu \nu}}{d R_{\mu \nu}}\right)_{0} \Delta R_{\mu \nu}+\sum_{\substack{\mu, \nu \\ \text { in } V}}\left(\frac{d^{2} E_{\mu \nu}}{d R_{\mu \nu}^{2}}\right)_{0}\left(\Delta R_{\mu \nu}\right)^{2}+\cdots
$$

Equation (4.12) is equivalent to (4.4) for central forces, and yields (4.10), from which we deduced the Cauchy relations.

To conform with the work of other authors, we introduce Cartesian coordinates $y^{i}$, and the displacement $s_{i}$. Distinction between covariant and contravariant components is now immaterial.

Using Cartesian coordinates $y_{\mu \nu}^{i}$, we have

$$
\begin{aligned}
\Delta R_{\mu \nu}=\sum_{i}\left(y_{\mu \nu}^{i}+s_{\mu \nu}^{i}\right)^{2}-\sum_{i}\left(y_{\mu \nu}^{i}\right)^{2} & =2 y_{\mu \nu}^{i} s_{i \mu \nu}+\sum_{i}\left(s_{i \mu \nu}\right)^{2}, \\
& =2 y_{\mu \nu}^{i} s_{i \mu \nu}+\delta^{i j} s_{i \mu \nu} s_{j \mu \nu} .
\end{aligned}
$$

We shall use the relations

$$
\begin{gathered}
\left(\frac{\partial E_{\mu \nu}}{\partial y_{\mu \nu}^{i}}\right)_{0}=\left(\frac{d E_{\mu \nu}}{d R_{\mu \nu}}\right)_{0} 2 y_{\mu \nu}^{i}, \\
\left(\frac{\partial^{2} E_{\mu \nu}}{\partial y_{\mu \nu}^{i} \partial y_{\mu \nu}^{i}}\right)_{0}=2 \delta^{i i}\left(\frac{d E_{\mu \nu}}{d R_{\mu \nu}}\right)_{0}+4 y_{\mu \nu}^{i} y_{\mu \nu}^{i}\left(\frac{d^{2} E_{\mu \nu}}{d R_{\mu \nu}^{2}}\right)_{0} .
\end{gathered}
$$

Substituting (4.13), (4.14), and (4.15) into (4.12), we get

$$
\begin{aligned}
E_{V} & =\frac{1}{2} \sum_{\substack{\mu, \nu \\
\text { in } V}}\left(\frac{\partial E_{\mu \nu}}{\partial y_{\mu \nu}^{i}}\right)_{0} s_{i \mu \nu}+\frac{1}{4} \sum_{\substack{\mu, \nu \\
\text { in } V}}\left(\frac{\partial^{2} E_{\mu \nu}}{\partial y_{\mu \nu}^{i} \partial y_{\mu \nu}^{i}}\right) s_{0} s_{i \mu \nu} s_{j \mu \nu}+\cdots, \\
& =V_{1}+V_{2}+\cdots,
\end{aligned}
$$

where summation is over all repeated indices, regardless of position. Equation (4.16) is exactly the formula used as a starting point by Born and Epstein.

Now, Born and Epstein both start from (4.16) and try to reach (4.10). Born is unable to do this correctly since he does not use the correct expression for $s_{i \mu \nu}$ in terms of $e_{i j}$.

Born substitutes in (4.16) the linear approximation $s_{i \mu \nu}=e_{i j} y_{\mu \nu}^{i}$ :

$$
E_{V} \cong \frac{1}{2} \sum_{\substack{\mu, \nu \\ \text { in } V}}\left(\frac{\partial E_{\mu \nu}}{\partial y_{\mu \nu}^{i}}\right)_{0} y_{\mu \nu}^{i} e_{i i}+\frac{1}{4} \sum_{\substack{\mu, \nu \\ \text { in } V}}\left(\frac{\partial^{2} E_{\mu \nu}}{\partial y_{\mu \nu}^{i} \partial y_{\mu \nu}^{i}}\right)_{0} e_{i k} e_{j h} y_{\mu \nu}^{k} y_{\mu \nu}^{h}+\cdots
$$


Using (4.14) and (4.15), he arrives at

$$
\begin{gathered}
E_{V} \cong \frac{1}{2} \sum_{\substack{\mu, \nu \\
\text { in } V}}\left(\frac{d E_{\mu \nu}}{d R_{\mu \nu}}\right)_{0} 2 y_{\mu \nu}^{i} y_{\mu \nu}^{i} e_{i i}+\frac{1}{4} \sum_{\substack{\mu, \nu \\
\text { in } V}}\left(\frac{d E_{\mu \nu}}{d R_{\mu \nu}}\right)_{0} 2 \delta^{i j} y_{\mu \nu}^{k} y_{\mu \nu}^{h} e_{i k} e_{i h} \\
+\frac{1}{4} \sum_{\substack{\mu, \nu \\
\text { in } V}}\left(\frac{d^{2} E_{\mu \nu}}{d R_{\mu \nu}^{2}}\right)_{0} 4 y_{\mu \nu}^{i} y_{\mu \nu}^{i} y_{\mu \nu}^{k} y_{\mu \nu}^{h} e_{i k} e_{i h}+\cdots
\end{gathered}
$$

By comparing (4.18) with (4.10), we see that Born's first order term is correct, but that his expression for the second order term is incorrect by a factor

$$
E_{2}^{\prime \prime}=\frac{1}{4} \sum_{\substack{\mu, \nu \\ i n V}} 2 \delta^{i i}\left(\frac{d E_{\mu \nu}}{d R_{\mu \nu}}\right)_{0} e_{i k} e_{i n} y_{\mu \nu}^{k} y_{\mu \nu}^{h}
$$

(see [2], p. 547, eqs. (31) and (32)).

Notice that (4.19) does not satisfy the Cauchy relations from a tensorial point of view. How then is Born able to deduce the Cauchy relations? He notices that, if the initial external forces are zero, then $E_{2}^{\prime \prime}=0$. Born actually never states explicitly that $E_{2}^{\prime \prime}=0$, when the initial external forces are zero, but he uses this result repeatedly to simplify his equations. Kellerman, a student of Born, does make the explicit statement that $E_{2}^{\prime \prime}=0$ and attributes it to Born (see [8], p. 534, note). In this case expression (4.18) reduces to the correct expression (4.10) up to terms of the second order (terms of higher order will always be incorrect). This is really a very fortunate coincidence, since it led Born to the correct conclusion.

In the case of initial external forces different from zero, Born would have arrived at the incorrect conclusion that $\Lambda^{i, h k}$ did not satisfy the Cauchy relations. Expressions (4.10) and (4.11) show that the Cauchy relations are satisfied even though the initial external forces be different from zero.

Let us now examine the work of Epstein. He considers only homogeneous strain for a monatomic crystalline substance (although he does not state this explicitly). He starts from (4.16), which he first rewrites in an unnecessarily complicated manner. He integrates approximately the partial differential equations connecting the strain and the displacements (1.9). His final formula for $s_{i \mu \nu}$ is:

$$
s_{i \mu \nu}=f_{l i} y_{\mu \nu}^{l}-\frac{1}{2} f_{l k} f_{j k} y_{\mu \nu}^{l},
$$

(see [4], eq. (14)) where $f_{i j}=e_{i j}+\omega_{i j}$ and $\omega_{i j}$ is the rotation

$$
\omega_{i i}=\frac{1}{2}\left(\frac{\partial s_{i \mu \nu}}{\partial y_{\mu \nu}^{i}}-\frac{\partial s_{i \mu \nu}}{\partial y_{\mu \nu}^{i}}\right) .
$$

(Born uses

$$
\left.s_{i \mu \nu}=e_{i j} y_{\mu \nu}^{i} .\right)
$$

Within the volume $V$, it is clear that $e_{i j}, \omega_{i i}$ are constant. He then substitutes (4.20) into $V_{1}$ in (4.16). In $V_{2}$ (the term quadratic in the displacements) he substitutes $s_{i \mu \nu}=$ $f_{l i} y_{\mu \nu}^{l}$, as Born does.

Upon substitution of (4.20) into $V_{1}$ we will get a term linear in $e_{i i}$, call it $V_{1}^{\prime}$, and a term quadratic in $e_{i j}$, call it $V_{1}^{\prime \prime}$. Epstein considers $V_{1}^{\prime \prime}+V_{2}$ (which is the whole term 
quadratic in $e_{i j}$, and which we have previously called $\varphi_{2}$ (energy density) or $E_{2}$ (energy)). He sets the coefficient of the expansion of $V$ with respect to the powers and products of $\omega_{i j}$ equal to zero. He then arrives at a number of rather complicated relations between the coefficients, which he uses to simplify the terms involving only $e_{i j}$. He then concludes, after this laborious task, that the coefficients of $\left[V_{1}^{\prime \prime}+V_{2}\right]\left(=E_{2}\right)$ satisfy relations of the Cauchy type. This we have proved in a simpler and more straightforward way.

He restricts himself to the case of zero initial external forces, and shows that $V_{1}=0$, hence that $E=V_{2}+\cdots$. If we neglect higher order terms, we have $E=V_{2} \cdot\left(V_{1}=0\right.$ since

$$
\sum_{\nu}\left(\frac{\partial E_{\mu \nu}}{\partial y_{\mu \nu}^{i}}\right)_{0}
$$

is the $i$ th component of the initial force on the particle $\mu=0$.)

He then has two quadratic forms in $e_{i i}$ at his disposal:

(a) $V_{1}^{\prime \prime}+V_{2}$, which satisfies the Cauchy relations $\left(=E_{2}\right)$, and

(b) $V_{2}$, which is the energy.

He now claims that the coefficients of elasticity appear in $V_{2}$ and not in $V_{1}^{\prime \prime}+V_{2}$, hence that the coefficients of elasticity do not satisfy the Cauchy relations. Actually the coefficients of elasticity (for the case of zero initial external forces) appear both in $V_{1}^{\prime \prime}+V_{2}$ and in $V_{2}$, since it is easy to show that $V_{1}^{\prime \prime}=0$.

We have that

$$
\phi=\Omega^{i j} e_{i i}+\Lambda^{i i, h k} e_{i j} e_{h k}+\cdots .
$$

Since the original state is stable, it is clear that $\Omega^{i i}=0$ for the case of zero external forces. This can be shown in another way, using a formula which Epstein takes as a basis for his argument:

$$
\tau^{i i}=\frac{\partial \phi}{\partial e_{i j}}=\Omega^{i i}+2 \Lambda^{i i, h k} e_{h k}+\cdots .
$$

If the original external forces are zero, then the initial stress is certainly zero; hence $\Omega^{i j}=0$. Consequently

$$
\phi=\phi_{2}+\cdots \quad \text { or } \quad E=E_{2}+\cdots .
$$

Limiting ourselves to terms of the second order in $e_{i j}$, we have that

$$
E=E_{2}=V_{1}^{\prime \prime}+V_{2} \text {. }
$$

But we also have that $E=V_{2}$. The only possible conclusion then is that $V_{1}^{\prime \prime}=0$. Formula (4.22) shows clearly that if any terminological distinction is to be made between the coefficients in $E_{2}$ and $V_{2}$, the coefficients of $E_{2}=V_{1}^{\prime \prime}+V_{2}$, and not the coefficients of $V_{2}$, must be called the coefficients of elasticity. In any event, the coefficients are equal and $E_{2}=V_{1}^{\prime \prime}+V_{2}$, represents the energy just as well as $V_{2}$.

The fact that $V_{1}^{\prime \prime}=0$ has been conclusively proven by Poincaré and Born (e.g. see [3], p. 45). It might be illuminating actually to substitute (4.20) and (4.21) in their respective positions into (4.16) and see that Epstein's formula for the energy is indeed correct (of course only up to second order terms, which is all he claims). Actually, Epstein goes about this in a more complicated way but with no apparent advantage. 
We have upon substitution:

$$
\begin{gathered}
E_{V}=\frac{1}{2} \sum_{\substack{\mu, \nu \\
\text { in } V}}\left(\frac{\partial E_{\mu \nu}}{\partial y_{\mu \nu}^{i}}\right)_{0} f_{l i} y_{\mu \nu}^{l}-\frac{1}{4} \sum_{\substack{\mu, \nu \\
\text { in } V}}\left(\frac{\partial E_{\mu \nu}}{\partial y_{\mu \nu}^{i}}\right)_{0} f_{l k} f_{i k} y_{\mu \nu}^{l} \\
+\sum_{\substack{\mu, \nu \\
\text { in } V}}\left(\frac{\partial^{2} E_{\mu \nu}}{\partial y_{\mu \nu}^{i} \partial y_{\mu \nu}^{i}}\right)_{0} f_{l i} f_{m i} y_{\mu \nu}^{l} y_{\mu \nu}^{m}+\cdots
\end{gathered}
$$

Using (4.14) and (4.15), (4.24) becomes

$$
\begin{aligned}
E_{V}=\frac{1}{2} \sum_{\substack{\mu, \nu \\
\text { in } V}}\left(\frac{d E_{\mu \nu}}{d R_{\mu \nu}}\right)_{0} 2 y_{\mu \nu}^{i} y_{\mu \nu}^{l} f_{l i}-\frac{1}{4} \sum_{\substack{\mu, \nu \\
\text { in } V}}\left(\frac{d E_{\mu \nu}}{d R_{\mu \nu}}\right)_{0} 2 y_{\mu \nu}^{i} y_{\mu \nu}^{l} f_{l k} f_{i k} \\
+\frac{1}{4} \sum_{\substack{\mu, \nu \\
\text { in } V}} 2 \delta^{i j}\left(\frac{d E_{\mu \nu}}{d R_{\mu \nu}}\right)_{0} y_{\mu \nu}^{l} y_{\mu \nu}^{m} f_{l i} f_{m i} \\
+\frac{1}{4} \sum_{\substack{\mu, \nu \nu \\
\text { in } V}}\left(\frac{d^{2} E_{\mu \nu}}{d R_{\mu \nu}^{2}}\right)_{0} 4 y_{\mu \nu}^{i} y_{\mu \nu}^{i} y_{\mu \nu}^{l} y_{\mu \nu}^{m} f_{l i} f_{m i}+\cdots
\end{aligned}
$$

or

$$
\begin{aligned}
E_{V}=\frac{1}{2} \sum_{\substack{\mu, \nu \\
\text { in } V}}\left(\frac{d E_{\mu \nu}}{d R_{\mu \nu}}\right)_{0} 2 y_{\mu \nu}^{i} y_{\mu \nu}^{l} f_{l i} \\
\quad+\frac{1}{4} \sum_{\substack{\mu, \nu \\
\text { in } V}}\left(\frac{d^{2} E_{\mu \nu}}{d R_{\mu \nu}^{2}}\right)_{0} 4 y_{\mu \nu}^{i} y_{\mu \nu}^{i} y_{\mu \nu}^{l} y_{\mu \nu}^{m} f_{l i} f_{m i}+\cdots .
\end{aligned}
$$

Setting all the coefficients of terms involving $\omega_{i j}$ in this equation equal to 0 , we get (4.10) up to second order terms:

$$
\begin{aligned}
E_{V}= & \frac{1}{2} \sum_{\substack{\mu, \nu \\
\text { in } V}}\left(\frac{d E_{\mu \nu}}{d R_{\mu \nu}}\right)_{0} 2 y_{\mu \nu}^{i} y_{\mu \nu}^{i} e_{i j} \\
& +\frac{1}{4} \sum_{\substack{\mu, \nu \\
\text { in } V}}\left(\frac{d^{2} E_{\mu \nu}}{d R_{\mu \nu}^{2}}\right)_{0} 4 y_{\mu \nu}^{i} y_{\mu \nu}^{i} y_{\mu \nu}^{h} y_{\mu \nu}^{k} e_{i j} e_{h k}+\cdots, \\
E_{V}= & E_{1}+E_{2}+\cdots .
\end{aligned}
$$

The quantity $E_{2}$ Epstein calls $V_{1}^{\prime \prime}+V_{2}$ because, in his derivation, $E_{2}$ stems partly from $V_{1}$ and partly from $V_{2}$. This has no theoretical significance. We can derive (see derivation (4.12) to (4.16)) the series in the displacements from the series in terms of the strain and find that $E_{1}$ yields $V_{1}$ and part of $V_{2}$. These statements are in no way contradictory. In any case, $E_{V}=E_{2}=V_{1}^{\prime \prime}+V_{2}=V_{2}$ (see (4.23)), where the initial external forces are zero. We have the expression for $V_{1}^{\prime \prime}$ in (4.25):

$$
V_{1}^{\prime \prime}=-\frac{1}{4} \sum_{\substack{\mu, \nu \\ i \mathrm{n} V}}\left(\frac{d E_{\mu \nu}}{d R_{\mu \nu}}\right)_{0} 2 y_{\mu \nu}^{i} y_{\mu \nu}^{i} e_{l k} e_{i k} .
$$


The crux of Epstein's argument is that $V_{1}^{\prime \prime} \neq 0$ (see [4], p. 919); he states that the only difference with Voigt is a term

$$
\sum_{\substack{\mu, \nu \\ \text { in } V}}\left(\frac{d E_{\mu \nu}}{d R_{\mu \nu}}\right)_{0}\left[\left(y_{\mu \nu}^{2}\right)^{2}+\left(y_{\mu \nu}^{3}\right)^{2}\right] .
$$

Epstein also says that this expression does not, in general, vanish. Yet it was proven by Poincaré ([3], p. 45) and by us that $V_{1}^{\prime \prime}=0$. Hence (see (4.28)), we have:

$$
\sum_{\substack{\mu, \nu \\ \text { in } V}}\left(\frac{d E_{\mu \nu}}{d R_{\mu \nu}}\right)_{0} 2 y_{\mu \nu}^{i} y_{\mu \nu}^{i}=0
$$

(zero initial external forces). This also proves (4.19) used by Born. Hence certainly $(4.29)=0$, and the entire argument of Epstein breaks down.

We have $E_{V}=E_{2}\left(=V_{1}^{\prime \prime}+V_{2}\right)$, and the coefficients of $E_{2}$ are the coefficients of elasticity and satisfy the Cauchy relations.

5. The multiatomic case. Let us consider a multiatomic, crystalline, elastic body. It can be thought of as a superposition of many simple monatomic lattices. The body can also be represented as the repetition of some fundamental cell (containing all the different types of particles!).

It has been shown by Born ([2], [6]) that, if the laws of force between the particles of different simple lattices are not the same, it is impossible to consider the body as strained in the same manner as a continuum. He shows that this latter assumption would lead to a violation of the equilibrium of the particles (see also Love [7], note $B, p p$. $620-627)$.

We can make a very reasonable assumption as to the state of deformation of the body without violating the equilibrium conditions and without abandoning the concept of strain as defined for a continuum. Each individual monatomic lattice is strained as a continuum, in the manner described in Sec. 4. In addition, each simple lattice is displaced with respect to the others.

Let $e_{i}$ be the strain of all lattices. Let us picture a frame of reference consisting of a continuum $C$, superimposed on the molecular body, and let this continuum be subject to the strain $e_{i j}$ experienced by each of the monatomic lattices. Every particle $\mu$ of the body will have an initially coinciding representation $\mu_{c}$ on $C$. The representation of each particle on $C$ will be displaced under the strain $e_{i j}$ by an amount $\vec{s}_{\mu_{c}}$. The final position of the representation $\mu_{c}$ of a particle will not, in general, coincide with the actual final position of the particle $\mu$, since each lattice is undergoing an additional displacement with respect to the continuum.

Let the displacement between the deformed positions of a particle and its representation on the continuum be $\vec{u}_{\mu}$. In a region which is small on a macroscopic scale, $\vec{u}_{\mu}$ will be the same for all particles $\mu$ belonging to the same monatomic lattice. If our body consists of $n$ different lattices, the deformation is completely specified by the $3 n+6$ local constants

$$
u_{(1) i}, u_{(2) i}, \cdots, u_{(m) i}, \cdots, u_{(n) i}, e_{i j} .
$$

We recall that the $u_{(m) i}$ were introduced to insure preservation of equilibrium. We expect the imposition of the equilibrium conditions to yield $u_{(m) i}$ as a function of the local strain $e_{i j}$. 
In any event, let us start our development as in Sec. 4 and notice the differences as we go along. For a monatomic lattice we expected an energy density of type (1.1), which was easily predictable. For a multiatomic body, we expect the energy function to depend at first on $e_{i i}, u_{(1) i}, \cdots, u_{(n) i}$. We write tentatively

$$
\begin{aligned}
\phi=\phi_{0} & +\Omega^{i i} e_{i j}+\Lambda^{i i, h k} e_{i j} e_{h k}+\cdots \\
& +\sum_{(m)} \psi_{(m)}^{i} u_{(m) i}+\sum_{(m),\left(m^{\prime}\right)} H_{(m)\left(m^{\prime}\right)}^{i j} u_{(m) i} u_{\left(m^{\prime}\right) i}+\cdots \\
& +\sum_{(m)} T_{(m)}^{i j, k} u_{(m) k} e_{i i}+\cdots
\end{aligned}
$$

We have that

$$
\psi_{(m)}^{i}=\left[\frac{\partial \phi}{\partial u_{(m) i}}\right]_{0}=0
$$

to satisfy the original equilibrium. For final equilibrium, we must have $\partial \phi / \partial u_{(m) i}=0$, which leads to:

$$
2 \sum_{\left(m^{\prime}\right)} H_{(m)\left(m^{\prime}\right)}^{i j} u_{\left(m^{\prime}\right) i}+T_{(m)}^{h k, i} e_{h k}=0 .
$$

We have $3 n$ linear equations in the $3 n$ unknowns $u_{(m) i}$. These equations are not independent. but still admit of a solution (see Born [2], p. 552). The solution will yield $u_{(m) i}$ as a linear function of all the strain components. We expect that

$$
u_{(m) i}=\pi_{(m) i}^{h k} e_{h k} .
$$

Substituting in (5.2) leads to

$$
2 \sum_{\left(m^{\prime}\right)} H_{(m)\left(m^{\prime}\right)}^{i j} \pi_{\left(m^{\prime}\right) i}^{h k} e_{h k}+T_{(m)}^{h k, i} e_{h k}=0 .
$$

Using (5.4) we simplify (5.1) to give

$$
\begin{aligned}
\phi=\Omega^{i i} e_{i i} & +\Lambda^{i i, h k} e_{i i} e_{h k}+\cdots \\
& -\sum_{(m),\left(m^{\prime}\right)} H_{(m)\left(m^{\prime}\right)}^{i j} \pi_{\left(m^{\prime}\right)}^{h k} e_{h k} e_{l p}+\cdots .
\end{aligned}
$$

The terms appearing in (5.5) will be evaluated a little later on. We will notice then that $\Lambda^{i j, h k}$ satisfy the Cauchy relations under the assumption of central forces. We will also observe that the first line in (5.5) is identical with the energy density based on an assumption of continuous strain.

The complete coefficient of the quadratic term in $e_{i j}$ will satisfy the Cauchy relations if, and only if,

$$
B^{h k, l_{p}}=\sum_{(m),\left(m^{\prime}\right)} H_{(m)\left(m^{\prime}\right)}^{i j} \pi_{(m) i}^{h k} \pi_{\left(m^{\prime}\right) i}^{l p}
$$

satisfies the Cauchy relations.

We recall that the Cauchy relations held in the monatomic case because $\Lambda^{i, n k}{ }^{*}$ could be written as

$$
\Lambda^{i i, h k}=\sum_{\mu, \nu} A_{\mu \nu} a_{\mu \nu}^{i} a_{\mu \nu}^{i} a_{\mu \nu}^{k} a_{\mu \nu}^{h} \quad\left(A_{\mu \nu}=\text { scalar }\right) .
$$


It is clear that the simplest tensor of the fourth rank satisfying the Cauchy relations is

$$
\chi^{i i, h k}=v^{i} v^{i} v^{h} v^{k}
$$

where $v^{i}$ is an arbitrary contravariant vector.

Let us try to make the most optimistic assumptions possible in order to obtain the Cauchy relations. Let us assume the form of $\pi_{(m) i}^{h k}$ most likely to yield a $B^{h k, l_{p}}$ satisfying the Cauchy relations. Clearly the most favorable assumption is

$$
\pi_{(m) i}^{h k}=t_{i(m)} t_{(m)}^{h} t_{(m)}^{k},
$$

where $t_{(m)}^{h}$ is a vector depending on $m$, i.e. different for different $m$. (Notice that $\pi_{(m) i}^{h k}$ is a quantity related to the $m$ th lattice, and must be assumed different for different $m$.) Let us assume for simplicity that

$$
H_{(m)\left(m^{\prime}\right)}^{i i}=\alpha_{(m)}^{i} \alpha_{\left(m^{\prime}\right)}^{i}
$$

then

$$
B^{h k, l p}=\sum_{(m),\left(m^{\prime}\right)} \alpha_{(m)}^{i} \alpha_{\left(m^{\prime}\right)}^{i} t_{i(m)} t_{j\left(m^{\prime}\right)} t_{(m)}^{h} t_{(m)}^{k} t_{\left(m^{\prime}\right)}^{l} t_{\left(m^{\prime}\right)}^{p}
$$

In addition let us assume that the summation over $(i, j)$ can be performed, to yield

$$
B^{h k, l p}=\text { (constant) } \sum_{(m),\left(m^{\prime}\right)} t_{(m)}^{h} t_{(m)}^{k} t_{\left(m^{\prime}\right)}^{l} t_{\left(m^{\prime}\right)}^{p} .
$$

By inspection of (5.12) we see that the Cauchy relations are not satisfied as soon as we have two or more types of particles. For, let us write

$$
B^{l p, h k}=\text { (constant) } \sum_{(m),\left(m^{\prime}\right)} t_{(m)}^{h} t_{(m)}^{p} t_{\left(m^{\prime}\right)}^{l} t_{\left(m^{\prime}\right)}^{k} .
$$

Then comparing (5.12) with (5.13) we see that the coefficients are not equal since $t_{(m)}^{i} \neq t_{\left(m^{\prime}\right)}^{i}$ at least for some values of $(m),\left(m^{\prime}\right)$. Hence the Cauchy relations must fail for a multiatomic body even though subject to central forces.

It may be of interest to develop in detail the expression for $\phi$ in the case of a multiatomic body, thereby verifying, step-by-step, the argument given previously in general terms. We then can compare our series for $\phi$ (or $E$ ) with the one used by Born. We will arrive at the conclusion that the series used by Born is incorrect in the part involving the strain terms exclusively, but correct in the terms in $u_{i(m)}$ and the mixed terms in $u_{i(m)} e_{h k}$.

We will limit ourselves to the case of central forces, and we start with formula (4.4), rewritten for central forces:

$$
E_{V}=\frac{1}{2} \sum_{\substack{\mu, \nu \\ \text { in } V}}\left(\frac{d E_{\mu \nu}}{d R_{\mu \nu}}\right)_{0} \Delta R_{\mu \nu}+\frac{1}{4} \sum_{\substack{\mu, \nu \\ \text { in } V}}\left(\frac{d^{2} E_{\mu \nu}}{d R_{\mu \nu}^{2}}\right)_{0}\left(\Delta R_{\mu \nu}\right)^{2}+\cdots .
$$

Unfortunately we cannot write, as we did before,

$$
\Delta R_{\mu \nu}=2 e_{i j} a_{\mu \nu}^{i} a_{\mu \nu}^{i} .
$$

Formula (5.15) holds only if $\mu, \nu$ belong to the same monatomic lattice. If $\mu$ and $\nu$ belong to different lattices, then, in addition to the strain $e_{i j}$, we must take into account the difference in the additional displacements $\vec{u}_{\mu}$ and $\vec{u}_{\nu}$. 
Let $\vec{s}_{\mu}$ be the actual displacement of the particle $\mu$. Let $\vec{s}_{\mu_{c}}$ be the displacement of the representation of $\mu$ on the continuum $C$. Then

$$
\vec{s}_{\mu}=\vec{s}_{\mu_{c}}+\vec{u}_{\mu}
$$

and

$$
\vec{s}_{\mu \nu}=\vec{s}_{\mu_{c} \nu_{c}}+\vec{u}_{\mu \nu}, \quad \text { where } \vec{u}_{\mu \nu}=\vec{u}_{\nu}-\vec{u}_{\mu} .
$$

Let $R_{\mu_{0} \nu_{0}}=\rho_{\mu_{0} \nu_{0}}^{2}$, where $\rho_{\mu_{0} \nu_{0}}$ is the initial distance between $\mu$ and $\nu$;

$R_{\mu_{c} \nu_{c}}=\rho_{\mu_{c} \nu_{0}}^{2}$, where $\rho_{\mu_{0} \nu_{0}}$ is the strained distance between the representations of $\mu$ and $\nu$ on the continuum $C$; and

$R_{\mu \nu}=\rho_{\mu \nu}^{2} \quad$ where $\rho_{\mu \nu}$ is the deformed distance between $\mu$ and $\nu$.

The following diagram may clarify the situation.

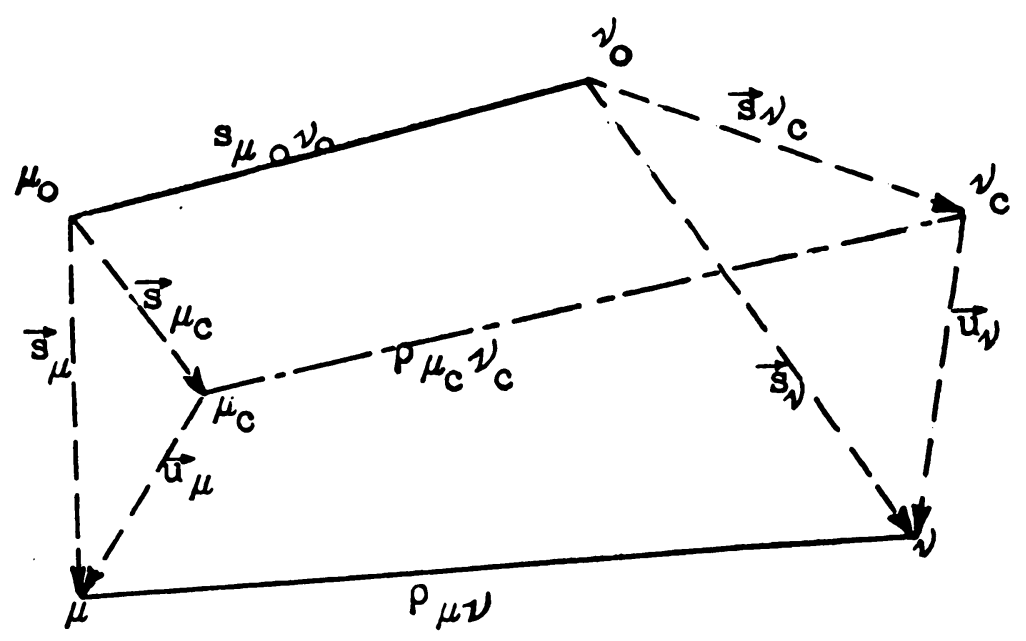

We have

$$
\Delta R_{\mu \nu}=R_{\mu \nu}-R_{\mu_{0} \nu_{0}},=R_{\mu \nu}-R_{\mu_{0} \nu_{0}}+R_{\mu_{0} \nu_{0}}-R_{\mu_{0} \nu_{0}},
$$

whence

$$
R_{\mu_{c} \nu_{0}}-R_{\mu_{0} \nu_{0}}=\left(\Delta R_{\mu \nu}\right)_{c}=2 e_{i j} a_{\mu \nu}^{i} a_{\mu \nu}^{i} .
$$

Clearly

$$
R_{\mu \nu}-R_{\mu_{c} \nu_{c}}=2\left(y_{\mu_{c} v_{c}}^{i}\right)\left(u_{\mu v i}\right)+\sum_{i}\left(u_{\mu v i}\right)^{2},
$$

where we are now using Cartesian coordinates.

Consequently,

$$
\Delta R_{\mu \nu}=\left(\Delta R_{\mu \nu}\right)_{c}+\delta^{i j} u_{\mu \nu i} u_{\mu \nu i}+2\left(y_{\mu \nu}^{i}+s_{\mu_{c} v}^{i}\right) u_{\mu \nu i}
$$

(since Cartesian coordinates are used, $s^{i}=s_{i}$, etc.), or

$$
\Delta R_{\mu \nu}=2 e_{i i} y_{\mu \nu}^{i} y_{\mu \nu}^{i}+\delta^{i j} u_{\mu \nu i} u_{\mu \nu i}+2 y_{\mu \nu}^{i} u_{\mu \nu i}+2 s_{\mu_{c \nu} c}^{i} u_{\mu \nu i} .
$$


Substituting in (5.16), we obtain

$$
\begin{aligned}
& E_{V}=\frac{1}{2} \sum_{\substack{\mu, \nu \\
\text { in } V}}\left(\frac{d E_{\mu \nu}}{d R_{\mu \nu}}\right)_{0} 2 e_{i i} y_{\mu \nu}^{i} y_{\mu \nu}^{i}+\frac{1}{4} \sum_{\substack{\mu, \nu \\
\text { in } V}}\left(\frac{d^{2} E_{\mu \nu}}{d R_{\mu \nu}^{2}}\right)_{0} 4 e_{i j} e_{h k} y_{\mu \nu}^{i} y_{\mu \nu}^{i} y_{\mu \nu}^{h} y_{\mu \nu}^{k}+\cdots \\
& +\frac{1}{2} \sum_{\substack{\mu, \nu \\
\text { in } V}}\left(\frac{d E_{\mu \nu}}{d R_{\mu \nu}}\right)_{0} 2 y_{\mu \nu}^{i} u_{\mu \nu i}+\frac{1}{2} \sum_{\substack{\mu, \nu \\
\text { in } V}}\left(\frac{d E_{\mu \nu}}{d R_{\mu \nu}}\right)_{0} \delta^{i j} u_{\mu \nu i} u_{\mu \nu i}+\frac{1}{4} \sum_{\substack{\mu, \nu \\
\text { in } V}}\left(\frac{d^{2} E_{\mu \nu}}{d R_{\mu \nu}^{2}}\right)_{0}^{2} 4 y_{\mu \nu}^{i} y_{\mu \nu}^{i} u_{\mu \nu i} u_{\mu \nu i}+\cdots \\
& +\frac{1}{4} \sum_{\substack{\mu, \nu \\
\text { in } V}} 8\left(\frac{d^{2} E_{\mu \nu}}{d R_{\mu \nu}^{2}}\right)_{0} e_{i i} u_{\mu \nu k} y_{\mu \nu}^{i} y_{\mu \nu}^{i} y_{\mu \nu}^{k}+\cdots \\
& +\frac{1}{2} \sum_{\substack{\mu, \nu \\
\text { in } V}}\left(\frac{d E_{\mu \nu}}{d R_{\mu \nu}}\right)_{0} 2 s_{\mu \nu \nu,}^{i} u_{\mu \nu i}+\cdots
\end{aligned}
$$

In (5.22) we have included all terms up to and including second order terms in $e_{i j}$ and $u_{\mu v i}$. The first line of (5.22) has the familiar appearance of the strain energy encountered in Sec. 4 (see (4.10)). It represents the energy resulting from the deformation of all the lattices, excluding the energy due to the displacements of the lattices with respect to each other. Using an obvious extension of the terminology already introduced, we will call the energy terms in the first line of $(5.22)$ by $E_{c}$.

Examining the other terms appearing in (5.22), we first notice, by investigating the condition for equilibrium, that

$$
\sum_{\substack{\mu, \nu, \\ \text { in } V}}\left(\frac{d E_{\mu \nu}}{d R_{\mu \nu}}\right)_{0} y_{\mu \nu}^{i} u_{\mu \nu i}=0 .
$$

We are next interested in the cross-terms involving $e_{i j} u_{\mu \nu k}$. The only direct term of this type appears on the third line of (5.22). A veiled term of this type, however, appears in the fourth line, which includes all cross-terms in $s^{i}{ }_{\mu_{c} \nu_{c}} u_{\mu \nu i}$. We know that $s_{\mu_{c} \nu_{c}}^{i}=$ $f\left(e_{i j}\right)$, and since we are interested only in the term linear in $e_{i i}$, we can safely substitute

$$
s_{\mu_{c \nu}}^{i}=e_{i j} y_{\mu \nu}^{i}=\delta^{i k} e_{k j} y_{\mu \nu}^{i}
$$

We are now in a position to write an expression equivalent to (5.1). We have not yet written $u_{\mu v i}$ in terms of $u_{(m) i}$, but this is only a simple algebraic step. We have

$$
\phi=\frac{1}{V}\left\{\begin{array}{c}
\frac{1}{2} \sum_{\substack{\mu, \nu \\
\text { in } V}}\left(\frac{d E_{\mu \nu}}{d R_{\mu \nu}}\right)_{0} 2 e_{i i} y_{\mu \nu}^{i} y_{\mu \nu}^{i}+\frac{1}{4} \sum_{\substack{\mu, \nu \\
\text { in } V}}\left(\frac{d^{2} E_{\mu \nu}}{d R_{\mu \nu}^{2}}\right)_{0} 4 e_{i j} e_{h k} y_{\mu \nu}^{i} y_{\mu \nu}^{i} y_{\mu \nu}^{h} y_{\mu \nu}^{k}+\cdots \\
+\frac{1}{2} \sum_{\substack{\mu, \nu \\
\text { in } V}}\left[\delta^{i i}\left(\frac{d E_{\mu \nu}}{d R_{\mu \nu}}\right)_{0}+2 y_{\mu \nu}^{i} y_{\mu \nu}^{i}\left(\frac{d^{2} E_{\mu \nu}}{d R_{\mu \nu}^{2}}\right)_{0}\right]_{\mu \nu i} u_{\mu \nu i}+\cdots \\
+\sum_{\substack{\mu, \nu \\
\text { in } V}}\left[\delta^{k i} y_{\mu \nu}^{i}\left(\frac{d E_{\mu \nu}}{d R_{\mu \nu}}\right)_{0}+2 y_{\mu \nu}^{i} y_{\mu \nu}^{i} y_{\mu \nu}^{k}\left(\frac{d^{2} E_{\mu \nu}}{d R_{\mu \nu}^{2}}\right)_{0}\right] e_{i j} u_{\mu \nu k}+\cdots
\end{array}\right\}
$$


By using relations (4.14), (4.15), we rewrite (5.24) as:

$$
\phi=\phi_{c}+\frac{1}{V}\left\{\begin{array}{c}
\frac{1}{4} \sum_{\substack{\mu, \nu \\
\text { in } V}}\left(\frac{\partial^{2} E_{\mu \nu}}{\partial y_{\mu \nu}^{i} \partial y_{\mu \nu}^{i}}\right)_{0} u_{\mu \nu i} u_{\mu \nu i}+\cdots \\
+\frac{1}{2} \sum_{\substack{\mu, \nu \\
\text { in } V}}\left(\frac{\partial^{2} E_{\mu \nu}}{\partial y_{\mu \nu}^{i} \partial y_{\mu \nu}^{i}}\right)_{0} y_{\mu \nu}^{i} e_{i j} u_{\mu \nu k}+\cdots
\end{array}\right\}
$$

We can arrive at (5.24) or (5.25) in a somewhat easier way by using the results for a monatomic body, and starting from a series in terms of the displacements:

$$
E_{V}=E_{0}+\frac{1}{2} \sum_{\substack{\mu, \nu \\ \text { in } V}}\left(\frac{\partial E_{\mu \nu}}{\partial y_{\mu \nu}^{i}}\right)_{0} s_{\mu \nu i}+\frac{1}{4} \sum_{\substack{\mu, \nu \\ \text { in } V}}\left(\frac{\partial^{2} E_{\mu \nu}}{\partial y_{\mu \nu}^{i} \partial y_{\mu \nu}^{i}}\right)_{0} s_{\mu \nu i} s_{\mu \nu i}+\cdots .
$$

With $E_{0}=0$, and $\vec{s}_{\mu}=\vec{s}_{\mu_{c}}+\vec{u}_{\mu}$,

$$
\phi=\frac{1}{V}\left\{\begin{array}{c}
\frac{1}{2} \sum_{\substack{\mu, \nu \\
\text { in } V}}\left(\frac{\partial E_{\mu \nu}}{\partial y_{\mu \nu}^{i}}\right)_{0} s_{\mu c \nu c i}+\frac{1}{4} \sum_{\substack{\mu, \nu \\
\text { in } V}}\left(\frac{\partial^{2} E_{\mu \nu}}{\partial y_{\mu \nu}^{i} \partial y_{\mu \nu}^{i}}\right)_{0} s_{\mu c \nu c i} s_{\mu c \nu c i}+\cdots \\
+\frac{1}{2} \sum_{\substack{\mu, \nu \\
\text { in } V}}\left(\frac{\partial E_{\mu \nu}}{\partial y_{\mu \nu}^{i}}\right)_{0} u_{\mu \nu i}+\frac{1}{4} \sum_{\substack{\mu, \nu \\
\text { in } V}}\left(\frac{\partial^{2} E_{\mu \nu}}{\partial y_{\mu \nu}^{i} \partial y_{\mu \nu}^{i}}\right)_{0} u_{\mu \nu i} u_{\mu \nu j}+\cdots \\
+\frac{1}{4} \sum_{\substack{\mu, \nu \\
\text { in } V}} 2\left(\frac{\partial^{2} E_{\mu \nu}}{\partial y_{\mu \nu}^{i} \partial y_{\mu \nu}^{i}}\right)_{0} s_{\mu c \nu i} u_{\mu \nu i}+\cdots
\end{array}\right\}
$$

Now (5.27) is equivalent to Born's series (see [2], p. 547, eq. (31)).

We notice that the first line of (5.27) is exactly of the type found in the monatomic case and is equivalent to what we have called $\phi_{c}=E_{c} / V$. In the third line of (5.27), we are allowed to substitute

$$
s_{\mu_{c \nu} v_{c} i}=e_{i j} y_{\mu \nu}^{i} .
$$

We notice that

$$
\sum_{\substack{\mu, \nu \\ \text { in } V}}\left(\frac{\partial E_{\mu \nu}}{\partial y_{\mu \nu}^{i}}\right)_{0} u_{\mu \nu i}=0
$$

and that (5.27) therefore becomes (5.25).

Born substitutes $s_{\mu_{c} v_{c i}}=e_{i} y_{\mu \nu}^{i}$ throughout (5.27); actually this substitution is only permissible in the third line of (5.27). Hence Born arrives at an incorrect expression for $E_{V}$ and $\phi_{c}$, but, since his error is localized in $\phi_{c}$, he is still able to deduce correctly the equations equivalent to (5.2) through (5.6), which are the salient equations of the multiatomic case. 


\section{APPENDIX}

We have written the total strain energy of a molecular body as

$$
\begin{aligned}
E & =\sum_{\mu, \nu}\left(\frac{\partial E}{\partial R_{\mu \nu}}\right)_{0} \Delta R_{\mu \nu}+\frac{1}{2} \sum_{\mu, \nu ; \eta, \xi}\left(\frac{\partial^{2} E}{\partial R_{\mu \nu} \partial R_{\eta \xi}}\right)_{0} \Delta R_{\mu \nu} \Delta R_{\eta \xi}+\cdots, \\
& =E_{1}+E_{2}+\cdots,
\end{aligned}
$$

where $\sum_{\mu, \nu}$ means a summation over all pairs of particles $\mu, \nu$.

We then defined the strain energy of a volume $V$ as:

$$
\begin{aligned}
E_{V} & =\sum_{\substack{\mu, \nu \\
\text { in } V}}\left(\frac{\partial E}{\partial R_{\mu \nu}}\right)_{0} \Delta R_{\mu \nu}+\sum_{\substack{\mu, \nu ; \eta, \xi \\
\text { in } V}}\left(\frac{\partial^{2} E}{\partial R_{\mu \nu} \partial R_{\eta \xi}}\right)_{0} \Delta R_{\mu \nu} \Delta R_{\eta \xi}+\cdots, \\
& =E_{V_{1}}+E_{V_{2}}+\cdots .
\end{aligned}
$$

If the body is divided into two parts $V$ and $V^{\prime}$, we notice that

$$
E_{V}+E_{V^{\prime}} \neq E \text {. }
$$

The reason for this inequality is physically clear. We have omitted the surface energy, i.e. the energy between the volumes $V$ and $V^{\prime}$. This energy is denoted by $E_{V V^{\prime}}$. It is our contention that $E_{V V^{\prime}}$ is negligible compared with $E_{V}$ and $E_{V}$, if the volumes $V, V^{\prime}$ are large compared with the sphere of molecular activity. Then the inequality (A.3) would become an equality:

$$
E_{V}+E_{V^{\prime}}=E .
$$

We will first show that $E_{V_{1}}+E_{V^{\prime}{ }_{1}}=E_{1}$, the arguments for higher order terms running similarly. We have exactly:

$$
E_{V 1}+E_{V 1^{\prime}}+E_{V 11^{\prime}}=E_{1} .
$$

We notice that $E_{V 1, V^{\prime} 1}$ consists of a summation over pairs of particles, one of which is situated in $V$, the other in $V^{\prime}$. The only pairs of particles, which will contribute appreciably to this summation, are those within an extremely narrow zone about the boundary of $V$ and $V^{\prime}$. The volume of this zone is of the order of the product of boundary surface multiplied by the range of appreciable molecular activity. This volume is therefore very small compared with either $V$ or $V^{\prime}$. Hence, it is permissible to write $E_{V 1}+E_{V^{\prime} 1}=$ $E_{1}$. The reasoning for $E_{2}$, etc. $\cdots$ is similar, and:

$$
E_{V}+E_{V^{\prime}}=E \text {. }
$$

If the body is split in more than two parts, we will still have

$$
\sum_{v, V^{\prime}, V^{\prime}, \ldots} E_{V}=E \text {. }
$$

The above argument is given in great detail by Poincaré ([3], pp. 40-53), and we have done little more than reproduce his exposition. (Note that long-range forces are not included in this treatment). 


\section{BIBLIOGRAPHY}

1. Brillouin, Les tenseurs en mécanique et en elasticite, Dover Publications, New York, 1946.

2. M. Born, Atomtheorie des Festen Zustandes, 2nd ed., B. G. Teubner, Leipzig, 1923.

3. H. Poincaré, Leçons sur la théorie de l'élasticité, Georges Carre, Paris, 1892.

4. P. S. Epstein, On the elastic properties of lattices, Phys. Rev. 70, 915-922 (1946).

5. W. Voigt, Lehrbuch der Kristallphysik, B. G. Teubner, Leipzig, 1910.

6. Handbuch der Physik, 2nd ed., Julius Springer, Berlin, 1933.

7. A. E. H. Love, $A$ treatise on the mathematical theory of elasticity, Dover Publications, New York, 1944.

8. E. W. Kellerman, The theory of the vibrations of the sodium chloride lattice, Phil. Trans. Roy. Soc. London (A) 238, 513-548 (1940). 